\title{
Fairness aware Chunk-Based Resource Allocation in Multi-Cell OFDMA Networks
}

\author{
Mahmoud M. Selim* ${ }^{*}$, Osamu Muta ${ }^{\dagger}$, Hossam Shalaby*, Hiroshi Furukawa ${ }^{\ddagger}$ \\ *Egypt-Japan University of Science and Technology (E-JUST), New Borg Al-Arab, Alexandria, Egypt \\ Email: mahmoud.sleem@ejust.edu.eg, shalaby@ieee.org \\ ${ }^{\dagger}$ Center for Japan-Egypt Cooperation in Science and Technology, Kyushu University, Fukuoka-shi, Fukuoka, Japan \\ Email: muta@ait.kyushu-u.ac.jp

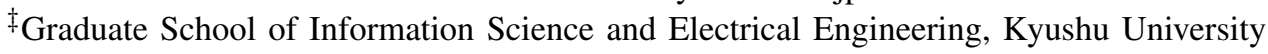 \\ Email: furuhiro@ait.kyushu-u.ac.jp
}

\begin{abstract}
Resource allocation (RA) in multi-cell OFDMA systems is very important for maximizing system throughput. Although sub-channel RA is optimal in terms of system throughput, more interest is given to chunk-based RA so as to simplify allocation algorithms and minimize required signalling. In this paper, we propose a fairness-aware chunk-based RA algorithm for the downlink transmission of multi-cell OFDMA system with fractional frequency reuse (FFR) adoption. Simulation results reveal that our proposed algorithm outperforms two reference algorithms in literature in terms of some system metrics such as average system spectral efficiency (SE), users fairness and rates of cell-edge users.
\end{abstract}

Index Terms-Resourec Allocation, Multi-Cell, Fairness, FFR, Chunk, OFDMA.

\section{INTRODUCTION}

Orthogonal frequency division multiple access (OFDMA) is a multiple access scheme currently exploited in most of modern wireless systems such as long term evolution-advanced (LTE-A) and IEEE 802.11/n to permit wide-band data services. OFDMA is immune against inter-symbol interference (ISI) by dividing wide-band frequency selective fading channel into orthogonal narrow-band flat fading sub-channels [1].

Fractional frequency reuse (FFR) is firstly proposed in [2] to solve the problem of strong co-channel interference encountered mainly by cell-edge users in multi-cell universal frequency reuse systems. FFR divides the macro-cell coverage area into two regions: center region with universal frequency reuse and edge region with reuse factor less than 1 (1/3 in our case).

Resource allocation (RA) problem in multi-cell OFDMA system is divided into three sub-problems; sub-channel allocation among users, power loading and bit loading on different sub-channels. These sub-problems should be jointly and efficiently solved with reasonable complexity. Many algorithms in the literature have been proposed to solve RA problem on a a sub-channel basis [3], [4] either to maximize system throughput or minimize transmitted power. RA on a subchannel basis has two main drawbacks: (1) RA algorithm complexity highly grows as number of sub-channels increases and (2) large signalling is required to be fed-back about channel information of each sub-channel. To simplify RA problem and reduce complexity, a number of contiguous subchannels are grouped together into one chunk and RA is done on a chunk basis rather than sub-channel basis.

Many algorithms in the literature have been proposed to solve the chunk-based RA problem in the single-cell scenario. Authors in [5] addressed system performance under many aspects such as fixed-size versus free-size chunks, equal power versus variable power per sub-channel and consecutive versus non-consecutive grouping using binary integer programming (BIP) models. Authors in [6] addressed the optimal chunkbased RA problem under bit error rate (BER) constraint considering the effect of dynamic power loading and coherence bandwidth on system throughput. Authors in [7] addressed chunk-based RA problem by dividing it into two separate subproblems (chunk assignment and power loading) to reduce complexity and simplify implementation. Some other works in the literature addressed chunk-based RA problem in MIMObased systems under BER constraint [8], with fairness guarantee [9] or under user rate constraint [10].

The main contribution of this paper is proposing a fairnessaware chunk-based RA algorithm for the downlink transmission of multi-user multi-cell OFDMA system with FFR adoption. We compare our proposed RA algorithm with two different algorithms in the literature in terms of average system spectral efficiency (SE), fairness among users and rates of celledge users. The first reference algorithm is called capacity maximization (CM) algorithm [11] in which RA is done in a two-step process. The first step is to allocate different chunks among users based on small scale fading channel conditions only ${ }^{1}$ such that each user is assigned the chunk with the highest channel condition. Power is then loaded homogeneously among chunks and bit loading is done so as to satisfy BER constraint on a further step. The second algorithm is the Round Robin (RR), the simplest allocation methodology, which allocates chunks among users regardless of their channel conditions. Although additional complexity is added by our proposed algorithm and SINR feedback is required at the transmitter ${ }^{2}$ compared to the two reference

\footnotetext{
${ }^{1}$ Authors assumed that this allocation methodology guarantees fairness

${ }^{2}$ modern wireless system such as LTE-A already includes SINR feed-back
} 


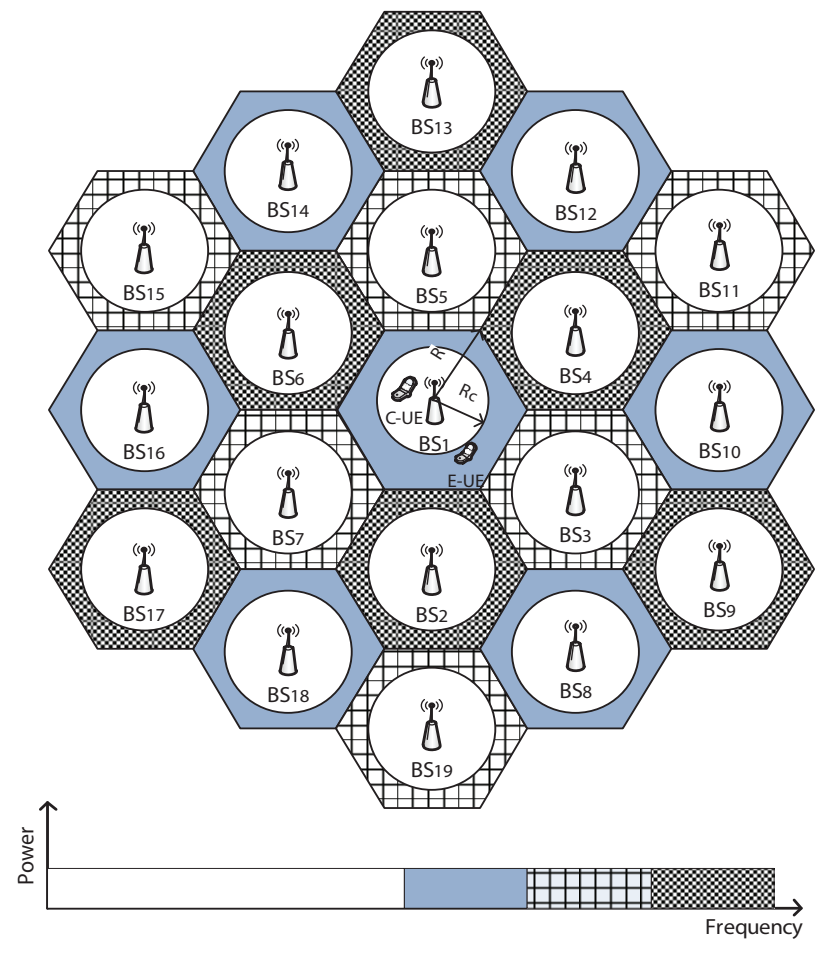

Fig. 1: Two-layer cellular system with FFR.

algorithms, this can be tolerated by the increase in system average SE and fairness among users.

The rest of the paper is organized as follows. System model is described in Section II. Our proposed chunk-based RA algorithm is described in Section III. Simulation and results are given in Section IV. The paper is concluded in Section V.

\section{SySTEM MODEL}

Our two-layer multi-cell OFDMA system consists of 19 macro-cells, each with radius $R$, as shown in Fig. 1. Each macro-cell is served by a single antenna macro base station $\left(B S_{i}\right), i=1,2, \ldots, 19$ located at the center of the cell $i$. Macro-cell coverage area is divided into two main regions; center region and edge region. Center region is bounded by a so-called FFR radius, $R_{c}$, from the center of the cell while edge region is located elsewhere. Two different reuse factors of 1 and $1 / 3$ are used for frequency sub-channels assigned for center user equipments (CUEs) and edge user equipments (EUEs) respectively such that frequency subchannels can be reused in areas with the same shadow as shown in Fig. 1. Total system bandwidth $B$ is divided equally into $N$ orthogonal narrowband flat fading sub-channels. $K$ active users, each with single receive antenna, are uniformly distributed within coverage area of the reference macro-cell $i=1$. If the user equipment (UE) is located inside center region of the reference macro-cell (i.e. CUE), strong cochannel interference will be generated from all neighbouring $B S_{i}, i=2,3, \ldots, 19$. If $\mathrm{UE}$ is located elsewhere (i.e. EUE), co-channel interference is generated from only a set of six BSs, $E, E=\{8,10,12,14,16,18\}$.
The normalized power frequency response of fading channel between any $B S_{i}$ and any user $k=1,2, \ldots, K$ over any subchannel $n, n=1,2, \ldots, N$ is given by

$$
h_{i, k, n}=\alpha_{i, k, n} . e^{j \phi_{i, k, n}}
$$

where $\alpha_{i, k, n}$ is the independently and identically Rayleigh distributed channel magnitude with unitary mean square, $E\left(\alpha_{i, k, n}^{2}\right)=1$, and $\phi_{i, k, n}$ is the channel phase with uniform distribution over $[0,2 \pi)$ for all $i, k$ and $n$. Coherence bandwidth of the channel is defined as $B_{c}=\frac{1}{2 \pi \sigma_{\tau}}$ where $\sigma_{\tau}$ is the rms delay spread. The correlation coefficient $\nu_{m, n}$ between any two sub-carriers $m$ and $n, m, n=1,2, \ldots, N$ over any fading channel is given by [12]

$$
\nu_{m, n}=E\left\{h_{i, k, m} h_{i, k, n}^{*}\right\}=\frac{1}{\sqrt{1+\frac{(m-n) \cdot \Delta f}{B_{c}}}}
$$

such that $(.)^{*}$ stands for complex conjugate and $\Delta f$ is subchannel bandwidth.

For chunk-based RA, $M$ contiguous sub-channels are grouped into one chunk, denoted as $c$, and allocation is done on a chunk basis. $C=\left\lfloor\frac{N}{M}\right\rfloor$ is the available number of chunks in the system where $\lfloor x\rfloor$ stands for the integer part of $x$. The normalized power frequency response vector associated with any user $k$ over any chunk $c, c=1,2, \ldots, C$ from $B S_{i}$ is $\mathbf{h}_{i, k, c}=\left[h_{i, k,(c-1) M+1}, \ldots, h_{i, k, c M}\right]$. The channel magnitude associated with any user $k$ over any chunk $c$, denoted as $\alpha_{i, k, c}$, is obtained by averaging over all sub-channels within chunk $c$ such that

$$
\alpha_{i, k, c}=\left(\frac{\mathbf{h}_{i, k, c} \mathbf{h}_{i, k, c}^{H}}{M}\right)^{\frac{1}{2}}
$$

where (. $)^{H}$ stands for conjugate transpose (Hermitian) and $\alpha_{i, k, c}$ is also independently and identically distributed for all $i, k$ and $c$ with unitary mean square, $E\left(\alpha_{i, k, c}^{2}\right)=1$. Both propagation path loss (PL) and log-normal shadowing are considered such that PL encountered between any $B S_{i}$ and any user $k$ is given by $d_{i, k}^{-\lambda}$ where $d_{i, k}$ is the distance from $B S_{i}$ to user $k$ in $m$ and $\lambda$ is the path loss exponent. Log normal shadowing $X_{\sigma}(\mathrm{dB})$ is also considered and identified as a random variable by its standard deviation, $\sigma(\mathrm{dB})$. Total encountered loss between $B S_{i}$ and user $k$, denoted as $g_{i, k}$, is given by

$$
g_{i, k}=d_{i, k}^{-\lambda} \cdot 10^{-X_{\sigma} / 10} .
$$

It has been proved in [6], [13] that dynamic power loading over chunks is not significant when small scale fading is only considered during chunk allocation and number of users is higher than two. Therefore, center region transmit power is equally allocated among center chunks and edge region transmit power is equally allocated among edge chunks subject to the total transmit power constraint $P_{\max }$. Moreover, it is assumed that all sub-channels within one chunk are allocated the same power. Therefore, transmitted power over any subchannel $n$ to any user $k$, denoted as $p_{k, n}$, is the same for all sub-channels that belongs to center chunk $c$ such that 
$p_{k, n}=p_{k,(c-1) M+1}=\ldots=p_{k, c M}=P_{\text {center. }}$. Similarly, transmitted power over any sub-channel $n$ to any user $k$, is the same for all sub-channels that belongs to edge chunk $c$ such that $p_{k, n}=p_{k,(c-1) M+1}=\ldots=p_{k, c M}=P_{\text {edge }}$. $P_{\text {center }}$ and $P_{\text {edge }}$ are sub-channel power of center and edge chunks respectively. This paper assumes that chunk allocation is globally known among all BSs, so that if a chunk is allocated to center region (or edge region) in the reference cell, it is allocated to center region (or edge region) in all other cells.

For any user $k$ located in the reference cell, power received on any sub-channel $n$ within chunk $c$ is given by

$$
P_{r}= \begin{cases}P_{\text {center }} \cdot \alpha_{1, k, c}^{2} \cdot g_{i, k} & \text { if user } k \text { is CUE } \\ P_{\text {edge }} \cdot \alpha_{1, k, c}^{2} \cdot g_{i, k} & \text { if user } k \text { is EUE }\end{cases}
$$

Co-channel interference generated from neighbouring cells on any user $k$ is defined as $I_{k}$ and depends on user location. For CUE, interference from 18 macro BSs, $\left(B S_{2} \sim B S_{19}\right)$, is encountered, while interference from a specific set of BSs, $E=\{8,10,12,14,16,18\}$, is experienced in case of EUE. $I_{k}$ is a random variable such that its variance, $\sigma_{I_{k}}^{2}$, is given by

$$
\begin{aligned}
\sigma_{I_{k}}^{2} & =\left\{\begin{array}{l}
E\left(\sum_{i=2}^{19} P_{c e n t e r} \cdot \alpha_{i, k, c}^{2} \cdot g_{i, k}\right), k \text { is CUE } \\
E\left(\sum_{i \in E} P_{\text {edge }} \cdot \alpha_{i, k, c}^{2} \cdot g_{i, k}\right), k \text { is EUE }
\end{array}\right. \\
& = \begin{cases}\sum_{i=2}^{19} P_{\text {center }} \cdot g_{i, k}, k \text { is CUE } \\
\sum_{i \in E} P_{\text {edge }} \cdot g_{i, k}, k \text { is EUE }\end{cases}
\end{aligned}
$$

Gaussian approximation in Eq.(6) is validated by the assumption that number of interferers for both types of users is larger than one. Therefore, average signal-to-interference plus noise power ratio (SINR) for any user $k$ over any chunk $c, \gamma_{k, c}$, is given by

$$
\begin{aligned}
& \gamma_{k, c}=\frac{P_{r}}{\sigma_{I_{k}}^{2}+\sigma_{\eta}^{2}}
\end{aligned}
$$

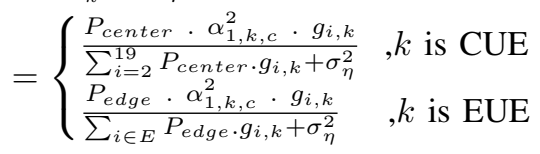

where $\sigma_{\eta}^{2}$ is the variance of thermal noise power at the receiver.

To simplify resource allocation, it is assumed that all subchannels within any chunk $c$ use the same bit loading. It is also assumed that adaptive $l$-ary quadrature amplitude modulation (QAM) is exploited to adopt with different channel conditions among chunks such that $l$ can be any of the set of values $L=$ $\{0,4,16,64\}$ and the corresponding number of transmitted bits per OFDM symbol, $b$, can be any of the set $B=\{0,2,4,6\}$. For $l$-ary QAM modulation, the BER encountered by any user $k$ on any sub-channel within chunk $c$ can be approximated in a closed form as [14]

$$
B E R_{k, c} \approx 0.2 \exp \left(\frac{c \cdot \gamma_{k, c}}{l_{k, c}-1}\right)
$$

where $c=-1.6$ and $l_{k, c}$ can be any value from the set $L$. Therefore, the $l$-ary QAM modulation exploited by any user $k$ over any sub-channel belongs to chunk $c$ under a BER constraint, $B E R_{t h}$, is given by

$$
l_{k, c}=\max _{l \in L}\left\{l \mid l \leq\left\lfloor 1+\frac{c \cdot \gamma_{k, c}}{\ln \left(5 B E R_{t h}\right)}\right\rfloor\right\}
$$

Then, the data rate transmitted on each sub-channel belongs to chunk $c$ by any user $k, r_{k, c}$, is given by

$$
r_{k, c}= \begin{cases}\log _{2} l_{k, c} & \text { if user } k \text { is CUE } \\ \log _{2}\left(l_{k, c}\right) / 3 & \text { if user } k \text { is EUE }\end{cases}
$$

The total rate achieved by any user $k, R_{k}$, is given by

$$
R_{k}=\sum_{c=1}^{C} a_{k, c} \cdot M \cdot r_{k, c}
$$

where $a_{k, c} \in\{0,1\}$ is a binary parameter to determine weather chunk $c$ is allocated to user $k\left(a_{k, c}=1\right)$ or not $\left(a_{k, c}=0\right)$. Finally, average system spectral efficiency per sub-channel, $S E$, is obtained by $S E=\frac{\sum_{k=1}^{K} R_{k}}{N}$

\section{Proposed Chunk-Based Resource Allocation ALGORITHM}

In this section, we propose a chunk-based RA algorithm with fairness provision for the downlink transmission of multiuser multi-cell OFDMA-based systems. During successive chunk allocation, our proposed algorithm employs the FFR concept to cope with co-channel interference and protect cell-edge users in the multi-cell scenario. The algorithm is described in details in Algorithm 1.

The proposed algorithm is initialized by zero rates $R_{k}=0$ for every user $k$ belongs to the set of all users $\psi$. The set of chunks $\Lambda$ is also initialized with null set $\varnothing$ on every chunk $c$. The first step of the algorithm is a round robin step such that, successively, each user $k \in \psi$ is allocated the chunk $c_{o p t}$ with the highest channel magnitude among set of remaining chunks $\Lambda$. If achievable rate by any user $k \in \psi$ is above zero, the chunk $c_{o p t}$ is assigned to user $k$ and removed from the set of available chunks $\Lambda$. Then, the total rate associated with user $k, R_{k}$, is also updated. Otherwise, the loop continues for the next user in the round robin step. This round robin step prevents starvation for users with poor channel conditions.

The next step is a fairness provision step such that the remaining set of chunks available in the set $\Lambda$ are fairly allocated among users. A set of candidate users $\psi_{\text {cand }}$ is initialized by the set of all users $\psi$ and the user $k_{\text {min }}=\underset{k \in \psi_{\text {cand }}}{\arg \min } R_{k}$ is selected so that fairness among users is implicitly enhanced. The chunk with the highest channel magnitude with respect to user $k_{\text {min }}$ among the set of chunks $\Lambda$, denoted as $c_{o p t}$, is selected for user $k_{\min }$. If the achievable rate by user $k_{\min }$ on the chunk $c_{o p t}$ is above zero, $c_{o p t}$ is assigned to user $k_{m i n}$, $R_{k_{m i n}}$ is updated and $c_{o p t}$ is removed from the set of chunks $\Lambda$. Otherwise, user $k_{\min }$ is removed from the set of candidate users $\psi_{\text {cand }}$ as it will not achieve rate over any other chunk as long as it doesn't achieve rate on its optimal chunk. The algorithm is terminated either if the set of candidate users 


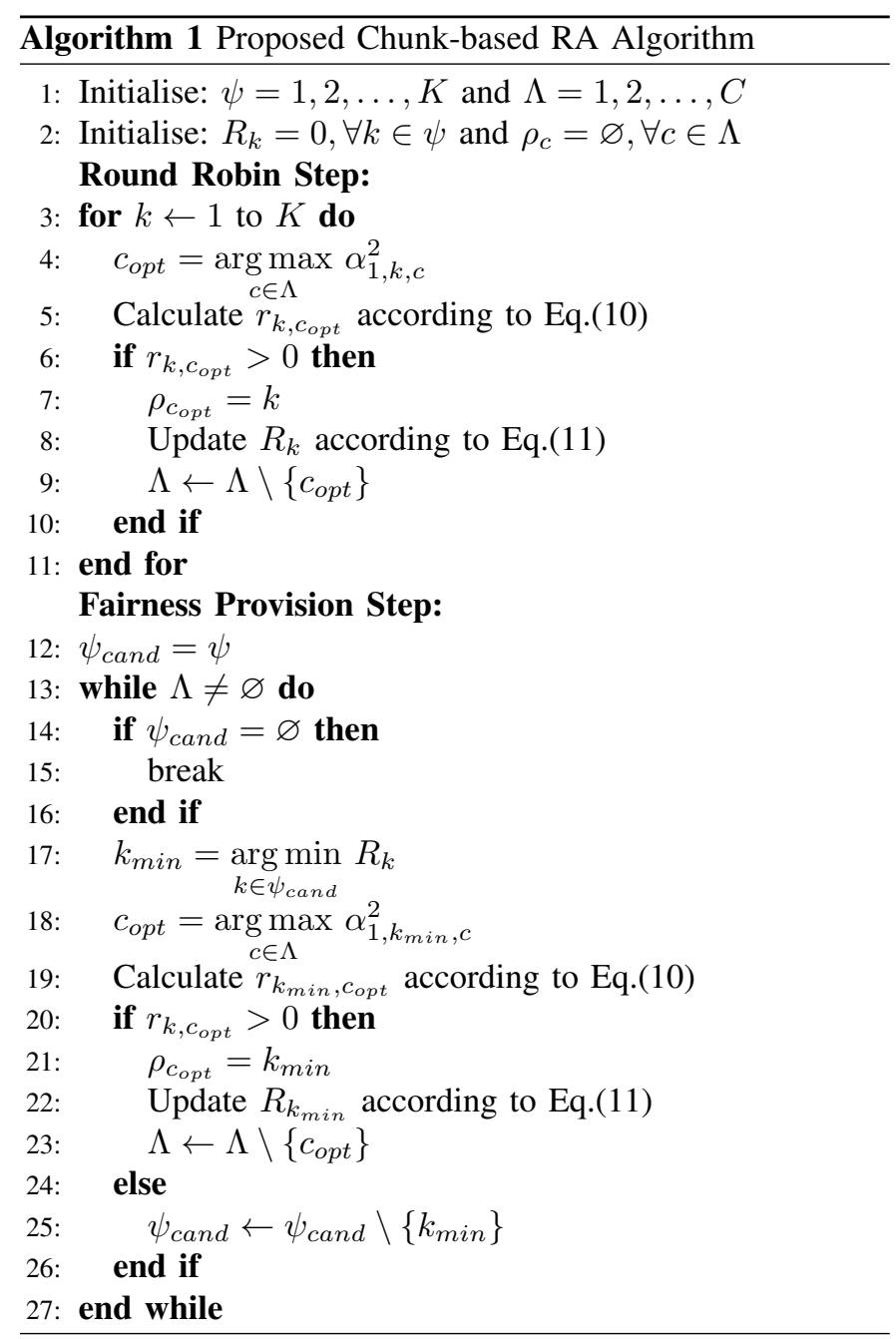

$\psi_{\text {cand }}$ becomes a null set (i.e. no user can achieve rate over any chunk of the remaining ones) or the set of chunks $\Lambda$ becomes a null set. If the set of available chunks $\Lambda$ is not empty at the algorithm termination, these chunks are considered in outage and unallocated. This fairness step maximizes system spectral efficiency while fairness among users is guaranteed as will be explained in the results section.

\section{Simulation \& Results}

In this section, we evaluate the performance of our proposed RA algorithm in terms of different system metrics compared to the reference algorithm in [11] and the RR algorithm. These metrics include average system spectral efficiency (SE), fairness index (FI) and rates of cell-edge users. Number of users, $K$, is set to 8 , average transmit signal to noise power ratio $S N R=\frac{P_{\text {center }}+P_{\text {edge }}}{\sigma_{\eta}^{2}}=20 d B$ and $P_{\text {center }}=P_{\text {edge }}$, number of sub-channels per chunk is set to $M=12$ and coherence bandwidth is $B_{c}=5 \Delta f$. Other simulation parameters are summarized in Table I. All results are obtained for $10^{4}$ channel realizations.

Fig. 2 shows average system SE per sub-channel against FFR radius ratio $R_{c} / R$ for the different algorithms. Results reveal that up to a specific radius ratio of 0.4 , capacity
TABLE I: System Parameters

\begin{tabular}{|l|l|}
\hline Parameter & Value \\
\hline Inter-site distance & $500 \mathrm{~m}$ \\
\hline Total Bandwidth & $100 \mathrm{MHz}$ \\
\hline Number of sub-channels N & 1024 \\
\hline Thermal noise power $\sigma_{\eta}^{2}$ & $-134 \mathrm{dBm} / \mathrm{Hz}$ \\
\hline Path-loss exponent $\lambda$ & 3 \\
\hline Shadow fading standard deviation $\sigma$ & $8 \mathrm{~dB}$ \\
\hline BER constraint & $10^{-3}$ \\
\hline
\end{tabular}

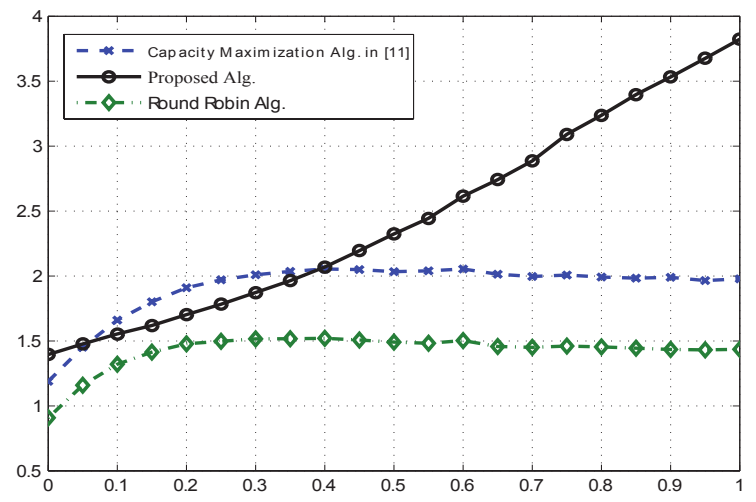

Fig. 2: Average SE per sub-channel vs. FFR ratio.

maximization (CM) algorithm has a better performance in terms of average SE due to low co-channel interference (CCI) generated from neighbouring cells. As FFR radius increases, center region area increases and therefore strong co-channel interference and path-loss are encountered by CUEs which limits the increase of achievable rates by CUEs as more chunks are considered in outage. Unlike CM algorithm, our proposed fairness-aware algorithm highly increases average system SE as shown in Fig. 2. These results can be explained directly from Fig. 3.

Fig. 3 shows the ratio of outage (unallocated) chunks at algorithm termination to total chunks against FFR radius ratio for the different algorithms. For CM algorithm, as FFR radius increases, more UEs are supposed to be in center area very far from serving BS and therefore encounter both very strong CCI and path-loss so more chunks are supposed to be in outage as shown in Fig. 3. Unlike CM algorithm, our proposed algorithm solves this problem by avoiding wasting chunks on users with very poor conditions due to either strong CCI or poor received signal strength. If any user fails to achieve rate on its optimal chunk, it will never achieve rate on any other chunk so it is useless to include it furthermore in chunk competition as previously explained in Algorithm 1. Fig. 3 shows clearly that number of wasted chunks in our proposed algorithm is very small compared to the two reference algorithms over the whole range of FFR radius.

Among different fairness measurements, we choose Jain's fairness index [15], defined as $F I=\frac{\left(\sum_{k} R_{k}\right)^{2}}{K \cdot \sum_{k} R_{k}^{2}}$, since it measures how fair or unfair the resources are shared among 


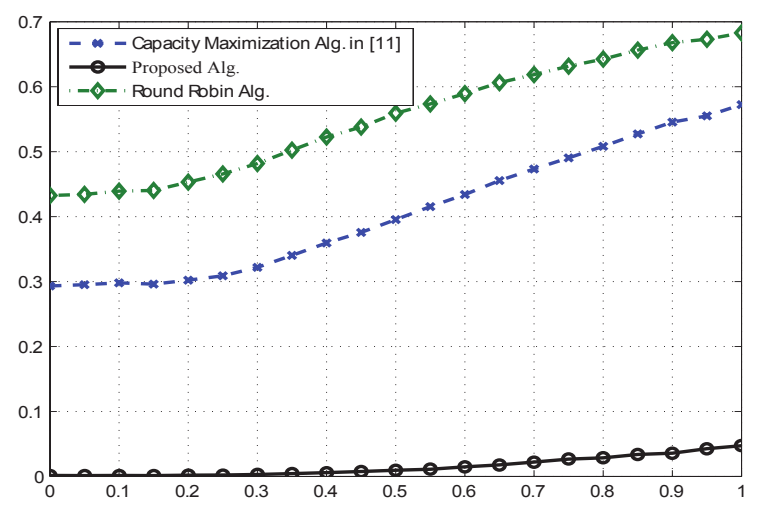

Fig. 3: Average ratio of outage chunks vs. FFR ratio.

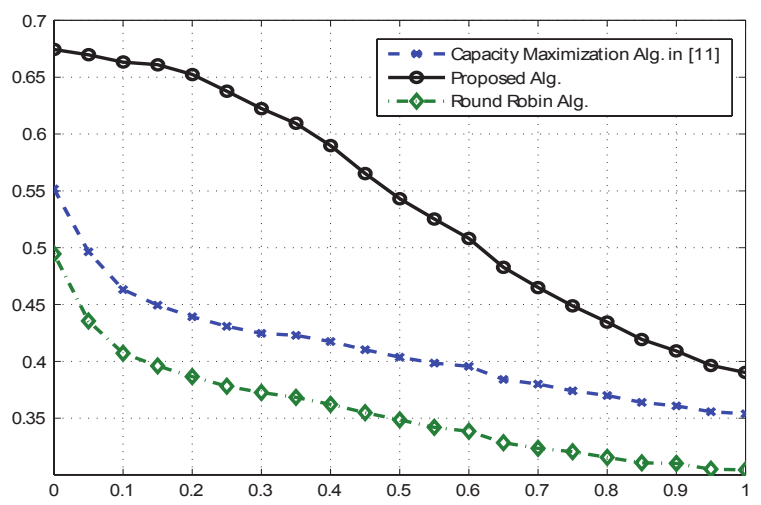

Fig. 4: Fairness index (FI) vs. FFR ratio.

users and ranges from $\frac{1}{K}$ (worst case) to 1 (best case). Fig. 4 shows the fairness index (FI) of different algorithms against FFR radius ratio. Our proposed algorithm highly increases fairness compared to the two reference algorithms by considering user with minimum rate during every chunk allocation in the fairness provision step. For comparison purpose, if we choose FFR radius ratio of 0.4 , at which our proposed algorithm and $\mathrm{CM}$ algorithm give the same average system SE, our proposed algorithm has fairness gain of about $50 \%$ over CM algorithm.

For further comparison of the different algorithms, cumulative distribution function (CDF) of rates per sub-channel associated with both all-users and cell-edge users are shown in Fig. 5 and 6 respectively at FFR radius ratio of 0.4. At FFR radius ratio of 0.4 , both our proposed algorithm and the $\mathrm{CM}$ algorithm have equal average SE required for fair comparison. It is clear from Fig. 5 that performance of both the CM and RR algorithms exceeds our proposed algorithm for only users with good conditions (near from BS). This is not the case for cell-edge users performance shown in Fig. 6. It is clear from Fig. 6 that our fairness-aware RA algorithm has enhanced performance of poor users at edge region by considering users with the minimum rate during every chunk allocation in the fairness provision step.

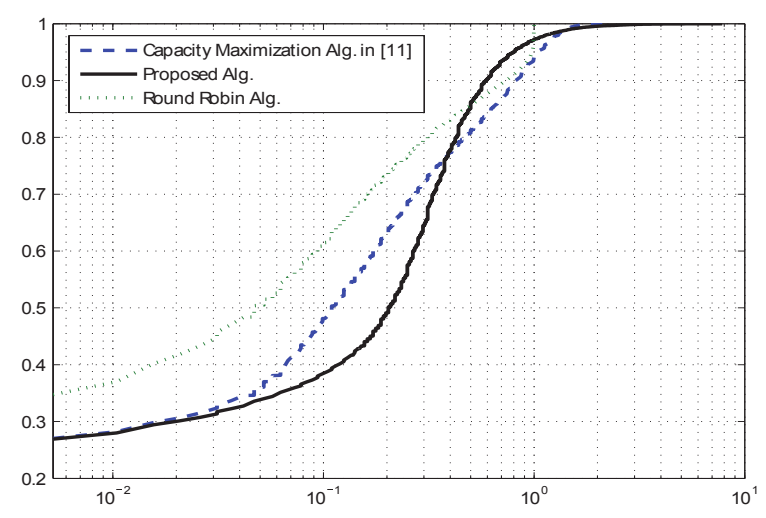

Fig. 5: CDF of all-user rates per sub-channel.

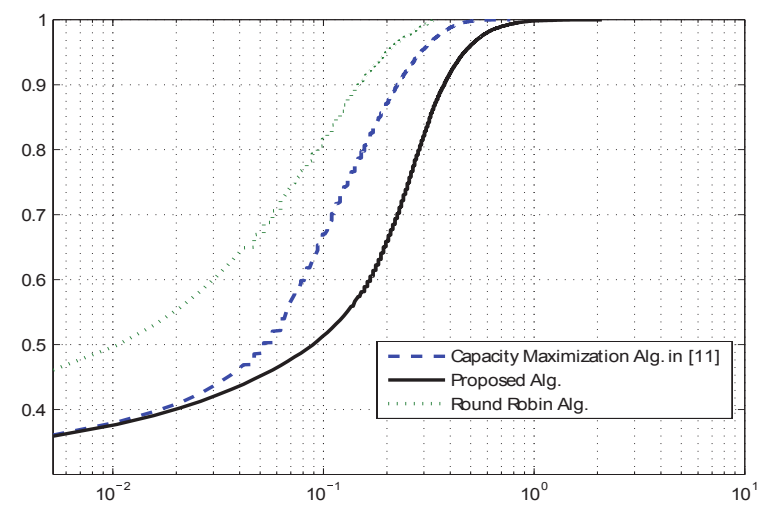

Fig. 6: CDF of cell-edge users rates per sub-channel.

\section{CONClusion}

In this paper, we proposed a fairness-aware chunk-based RA algorithm for the downlink transmission of multi-user multicell OFDMA systems with FFR adoption. Simulation results reveal that our proposed algorithm performance highly exceeds two reference algorithms in the literature in terms of average system SE by avoiding wasting resources on users with very poor conditions. Both total fairness among users and cell-edge users performance have been also enhanced due to considering users with the minimum rate during every chunk allocation in the fairness provision step. Although additional complexity is added by the proposed algorithm, it can be tolerated by the increase in system fairness and SE.

\section{REFERENCES}

[1] 3GPP, "Requirements for further advancements for E-UTRA (LTEAdvanced)," 3rd Generation Partnership Project (3GPP), TR 36.913, Jun. 2008. [Online]. Available: http://www.3gpp.org/ftp/Specs/htmlinfo/36913.htm

[2] R1-050507, "Soft frequency reuse scheme for UTRAN LTE," Tech. Rep. 3GPP TSG-RAN WG1 Meeting 41, May 2005.

[3] M. M. Selim, S. Elnoubi, H. Shalaby, O. Muta, and H. Furukawa, "Reduced complexity QoS aware resource allocation technique for MISOOFDMA systems," in Computing, Networking and Communications (ICNC), 2014 International Conference on. IEEE, 2014, pp. 950-954. 
[4] M. M. Selim, O. Muta, H. M. Shalaby, and H. Furukawa, "Low complexity QoS-aware subcarrier allocation for MISO-OFDMA systems," IEICE Communications Express, vol. 3, no. 8, pp. 235-240, 2014.

[5] L. Lei, V. Angelakis, and D. Yuan, "Performance analysis of chunkbased resource allocation in wireless OFDMA systems," in Computer Aided Modeling and Design of Communication Links and Networks (CAMAD), 2012 IEEE 17th International Workshop on. IEEE, 2012, pp. 90-94.

[6] H. Zhu and J. Wang, "Chunk-based resource allocation in OFDMA systems - part II : joint chunk, power and bit allocation," Communications, IEEE Transactions on, vol. 60, no. 2, pp. 499-509, 2012.

[7] J. Fan, Q. Yin, G. Y. Li, B. Peng, and X. Zhu, "Adaptive block-level resource allocation in OFDMA networks," Wireless Communications, IEEE Transactions on, vol. 10, no. 11, pp. 3966-3972, 2011.

[8] V. D. Papoutsis and A. P. Stamouli, "Chunk-based resource allocation in multicast MISO-OFDMA with average BER constraint." IEEE Communications Letters, vol. 17, no. 2, pp. 317-320, 2013.

[9] V. D. Papoutsis and S. A. Kotsopoulos, "Chunk-based resource allocation in distributed MISO-OFDMA systems with fairness guarantee," Communications Letters, IEEE, vol. 15, no. 4, pp. 377-379, 2011.

[10] P.-C. Chou, C.-Y. Ma, and C.-C. Huang, "Chunk-based resource allocation under user rate constraints in multiuser MIMO-OFDM systems," in ITS Telecommunications (ITST), 2012 12th International Conference on. IEEE, 2012, pp. 857-861.

[11] H. Zhu and J. Wang, "Frequency reuse in chunk-based multi-cell OFDMA systems," in Communications (ICC), 2012 IEEE International Conference on. IEEE, 2012, pp. 4668-4672.

[12] R. Prasad, OFDM for wireless communications systems. Artech House, 2004.

[13] S. H. Ali and V. C. Leung, "Dynamic frequency allocation in fractional frequency reused OFDMA networks," Wireless Communications, IEEE Transactions on, vol. 8, no. 8, pp. 4286-4295, 2009.

[14] A. J. Goldsmith and S.-G. Chua, "Variable-rate variable-power M-QAM for fading channels," IEEE Transactions on Communications, vol. 45, no. 10, pp. 1218-1230, 1997.

[15] R. Jain, D.-M. Chiu, and W. R. Hawe, A quantitative measure of fairness and discrimination for resource allocation in shared computer system. Eastern Research Laboratory, Digital Equipment Corporation, 1984. 